\title{
The continuum energy distribution of single galactic Wolf-Rayet stars
}

\author{
Bas A. den Hond ${ }^{1}$, Peter S. Conti ${ }^{2}$, and H. Lamers ${ }^{1}$ \\ ${ }^{1}$ Astronomical Institute, Utrecht University, the Netherlands \\ ${ }^{2} J I L A$, University of Colorado, Boulder CO, USA
}

\begin{abstract}
For eleven single galactic WR stars, the continuum energy distribution from the UV to the radio was obtained from literature data. Although a power law with $\alpha=2.79$ could describe all the distributions, there appear to be deviations that depend on stellar type.
\end{abstract}

\section{Introduction}

The spectral energy distribution remains an important diagnostic of the structure of optically thick stellar winds. For radio and IR, the approximation to the theoretical power law distribution for a non-accelerating wind (Barlow \& Wright 1975) is well established except for non-thermal effects. The UV and optical also show a power law $F_{\lambda} \propto \lambda^{-\alpha}$ with $\alpha \simeq 2.8$. Morris et al. (1993) used this to establish accurate color excesses of single galactic, LMC and SMC stars by 'nulling' the $2200 \AA$ absorption dip. In this study, results from different studies are combined to obtain the energy distribution over as large a baseline as possible.

\section{Data}

UV and optical continuum data were graciously provided by P.W. Morris and K.R. Brownsberger. Radio data were taken from the literature. Infrared data were taken from the Catalog of Infrared Observations by Gezari et al. (1997).

\section{Results}

The approach of Morris et al. was replicated with an extended baseline. Powerlaw behaviour remained the obvious description. Although extending the baseline resulted in fewer stars with data all over the baseline, the $\alpha$ parameters kept the approximately Gaussian distribution and insensitivity to stellar type found in that study. However, the spectral index often changed markedly as the baseline extended past 6 micron. At the full baseline, $1500 \AA$ to $20 \mathrm{~cm}$, eleven stars were left, with a mean $\alpha=2.79(\sigma=0.05)$.

A better fit is obtained when $\alpha$ is allowed to change somewhere in the infrared (6 micron was adopted). Due to this additional free parameter, the spectral index for $\lambda>6$ micron is seen to increase by a few tenths for early type stars, 
and decrease for late type stars. This behaviour is shown by both WC and WN stars. Details will be given in a forthcoming paper.

Acknowledgments. BAdH would like to thank JILA in Boulder for its hospitality in 1997, and the SOC, the Olga Koning Fonds and the Kapteyn Fonds for financial support.

\section{References}

Morris, P.W., Brownsberger, K.R., Conti, P.S., Massey, P., Vacca, W.D. 1993, ApJ 412, 324

Gezari, D.Y., Pitts, P.S., Schmitz, M. 1998, Catalog of Infrared Observations, May 1998 (v4.1), iuewww.gsfc.nasa.gov/cio/cio homepage.html

Wright, A.E., Barlow, M.J. 1975, MNRAS 170,41

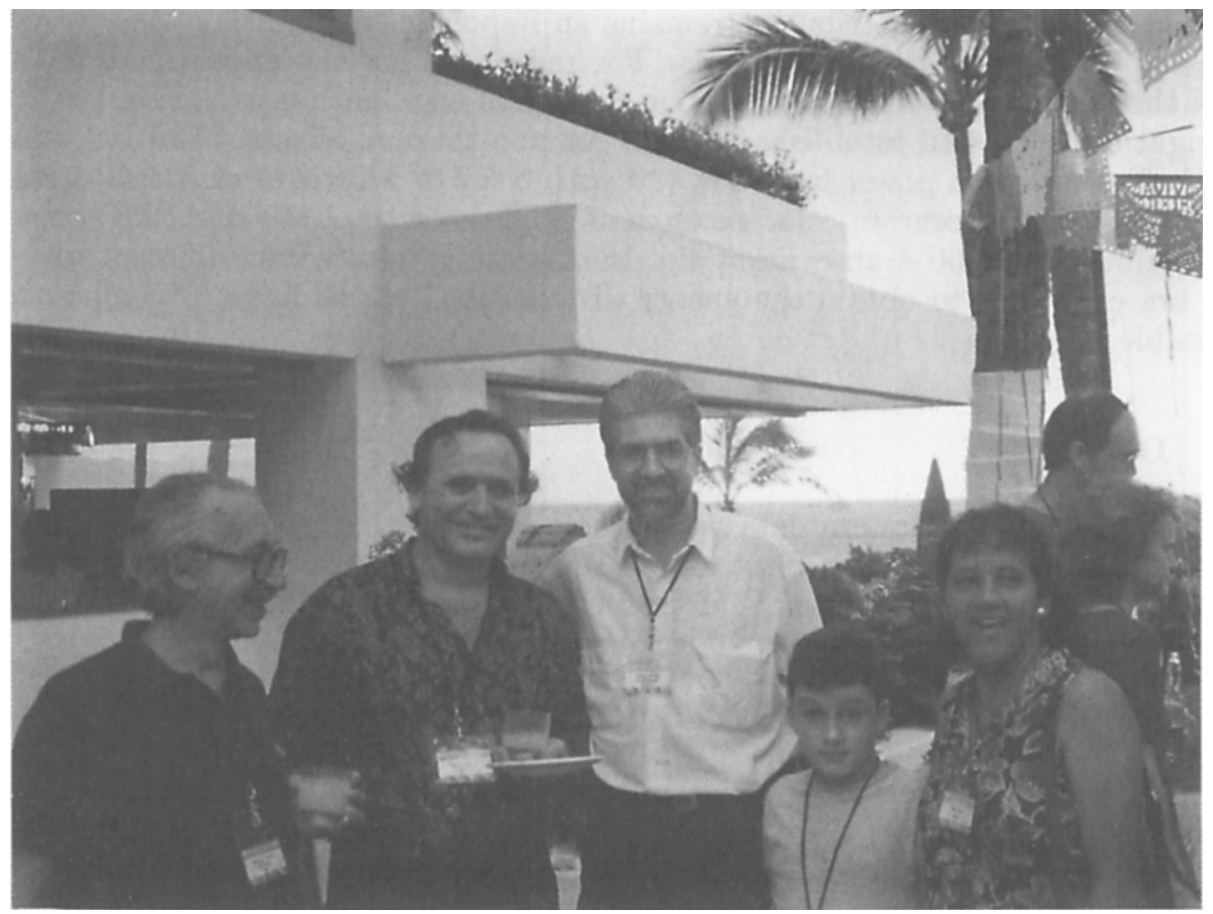

